\title{
THE INTEGRAL CLOSURE OF A NOETHERIAN RING
}

\author{
BY \\ JAMES A. HUCKABA $\left({ }^{1}\right)$
}

\begin{abstract}
Let $R$ be a commutative ring with identity and let $R^{\prime}$ denote the integral closure of $R$ in its total quotient ring. The basic question that this paper is concerned with is: What finiteness conditions does the integral closure of a Noetherian ring $R$ possess? Unlike the integral domain case, it is possible to construct a Noetherian ring $R$ of any positive Krull dimension such that $R^{\prime}$ is non-Noetherian. It is shown that if $\operatorname{dim} R<2$, then every regular ideal of $R^{\prime}$ is finitely generated. This generalizes the situation that occurs in the integral domain case. In particular, it generalizes Nagata's Theorem for two-dimensional Noetherian domains.
\end{abstract}

1. Introduction. A ring means a commutative ring with identity that is not necessarily an integral domain. If $R$ is a ring, then $T(R)$ is its total quotient ring and $R^{\prime}$ denotes its integral closure in $T(R)$. An overring of $R$ is a ring between $R$ and $T(R)$. An element in $R$ is regular if it is not a divisor of zero. A regular ideal is an ideal that contains a regular element.

A well-known result for integral domains is:

THEOREM A ([8, (33.2) and (33.12)]). If $D$ is a Noetherian domain of dimension $\leqslant 2$, then $D^{\prime}$ is a Noetherian domain.

For a history of this theorem, see the Historial Note Appendix of M. Nagata's book [8]. Our purpose is to give a generalization of Theorem $A$ to arbitrary commutative rings. We show at the end of $\$ 3$ how to construct a Noetherian ring $R$, of any positive dimension such that $R^{\prime}$ is a non-Noetherian ring. Hence, there is no possibility of Theorem A remaining true when "domain" is replaced by "ring". However, we are able to generalize Theorem A to the ring theory case as follows:

THEOREM B. If $R$ is a Noetherian ring such that $\operatorname{dim} R \leqslant 2$, then every regular ideal of $R^{\prime}$ is finitely generated.

If $\operatorname{dim} R=1$, then the proof of Theorem B is readily proved by reducing

Received by the editors May 14, 1975.

AMS (MOS) subject classifications (1970). Primary 13B20, 13E05; Secondary 13A15.

Key words and phrases. Integral closure, Noetherian ring, valuation ring, Krull dimension of a ring.

(1) This work was partially supported by a summer research grant at the University of Missouri.

Copyright $\odot$ 1976, American Mathematical Society 
to the integral domain case. However, for $\operatorname{dim} R=2$ the proof is much more difficult. Nagata's proof of the two-dimensional case for domains makes use of a reduction to the one-dimensional case by first factoring out of $R^{\prime}[X]$ a certain principal ideal that is a prime ideal of the Krull domain $R^{\prime}[X]$. The problems with the ring theory case when passing from $R^{\prime}$ to $R^{\prime}[X]$ are: (1) integral closure is not preserved; and (2) many new nilpotent elements are introduced. These new nilpotents are difficult to control. To overcome these problems we make use of a generalization of Krull domain introduced by J. Marot [7] and some recent results by L. J. Ratliff [9], [10]. Ratliff's results concern prime divisors of principal ideals for a certain class of rings of which $R, R^{\prime}$, and $R^{\prime}[X]$ are members.

$\$ 2$ contains Marot's definition of Krull rings and the major facts about these rings. $\$ 3$ is devoted entirely to the proof of the main theorem of this article-the proof of Theorem B.

The author is indebted to George Hinkle for Step 4 in the proof of Theorem B.

2. Krull rings. This section develops the theory of Krull rings. Even though these rings are quite interesting themselves, we will use them only as a tool to establish Theorem B.

A ring $R$ is called an additively regular ring if for each $x \in T(R)$ there exists $u \in R$ such that $x+u$ is a regular element of $T(R)$; see [3] and [4]. Noetherian rings and overrings of Noetherian rings are additively regular $[1, \mathrm{Lem}$ ma B]. Every regular ideal of an additively regular ring is generated by its set of regular elements [7, Proposition 1.1.2].

Let $T$ be a total quotient ring and let $\{G,+\}$ be a totally ordered abelian group. A map $v$ from $T$ onto $G \cup\{\infty\}$ is a valuation, if for all $x, y \in T$ :

(1) $v(x y)=v(x)+v(y)$

(2) $v(x+y) \geqslant \min \{v(x), v(y)\}$;

(3) $v(1)=0$ and $v(0)=\infty$.

The ring $V=\{x \in T: v(x) \geqslant 0\}$ is called the valuation ring of $v$. A valuation ring $V$ (resp., valuation $v$ ) is a discrete rank one valuation ring (resp., discrete rank one valuation), if the group $G$ is isomorphic to the group of integers.

Definition 2.1. A ring $R$ is a Krull ring if there exists a family $\left\{v_{i}\right\}$ of discrete rank one valuations such that:

(1) $R$ is the intersection of the corresponding valuation rings $\left\{V_{i}\right\}$

(2) for each regular $x \in T(R), v_{i}(x)=0$ for all but a finite number of $v_{i}$.

The idea of a valuation ring that contains zero divisors has been explored by many authors. General results about such rings may be found in [6]. Our definition of Krull ring is a slight generalization of Marot's definition [7, p. 27]. 
It agrees with the definition of Krull domain, when $R$ is assumed to be an integral domain.

Ratliff [9] considers the following class of rings, which we denote by $\mathcal{S}: R$ has only finitely many minimal prime ideals and the integral closure of $R / Z$ is a Krull domain, for each minimal prime ideal $Z$ of $R$. If $R$ is in $\mathcal{S}$, then every ring between $R$ and $R^{\prime}$ is in $\mathfrak{C}[9$, p. 212]. Every Noetherian ring is in $\mathfrak{C}$.

THEOREM 2.2. If $R$ is an additively regular member of $\mathfrak{S}$, then $R^{\prime}$ is a Krull ring.

Proof. Part 1. Assume that $R$ is a reduced ring and that $\left\{Z_{i}\right\}_{i=1}^{g}$ is the set of minimal prime ideals of $R$. Then, $N=\bigcap Z_{i}=(0)$. If $K_{i}$ is the quotient field of the domain $R / Z_{i}$, then $T(R)$ is the direct sum of the fields $\left\{K_{i}\right\}_{i=1}^{g}$ and $R^{\prime}$ is the direct sum of $\left\{\left(R / Z_{i}\right)^{\prime}\right\}_{i=1}^{g}$. Since $R$ is in $\mathbb{C},\left(R / Z_{i}\right)^{\prime}$ is a Krull domain. Write $\left(R / Z_{i}\right)^{\prime}=\bigcap V_{i j}$, where $\left\{V_{i j}\right\}_{j \in J}$ is the defining family of discrete rank one valuation domains for $\left(R / Z_{i}\right)^{\prime}$. Define $W_{i j}$ to be the direct sum

$$
K_{1} \oplus \cdots \oplus K_{i-1} \oplus V_{i j} \oplus K_{i+1} \oplus \cdots \oplus K_{g}
$$

Each $W_{i j}$ is a discrete rank one valuation ring. If $w_{i j}$ is the valuation associated with $W_{i j}$, then $\left\{w_{i j}\right\}$ satisfies Definition 2.1 for $R^{\prime}$. Therefore, $R^{\prime}$ is a Krull ring.

Part 2. Suppose that the nilradical $N$ of $R$ is nonzero. If $N^{\prime}$ denotes the nilradical of $R^{\prime}$, we may assume without loss of generality that:

(1) $R / N \subset R^{\prime} / N^{\prime} \subset(R / N)^{\prime} \subset T(R / N)=T\left(R^{\prime} / N^{\prime}\right)$;

(2) $T(R) / N^{\prime}=T(R) / N^{\prime} T(R) \subset T\left(R^{\prime} / N^{\prime}\right)$.

Since $R / N$ has only a finite number of maximal prime divisors of zero, \{namely, $Z_{i}$ (modulo $N$ ), $i=1,2, \ldots, g$ \}, $R / N$ is an additively regular ring [1, Lemma B] belonging to $[9$, p. 212]. By Part 1 , there is a family of discrete rank one valuations $\left\{v_{i}\right\}$, with corresponding valuation rings $\left\{V_{i}\right\}$, such that $(R / N)^{\prime}=\bigcap V_{i}$.

Let $V$ be one of the $V_{i}$ and let $I$ be the additive group of integers. In view of relation (2), we can define a mapping $w: T(R) \rightarrow I \cup\{\infty\}$ via $w(x)=$ $v\left(x+N^{\prime}\right)$. The map $w$ is a valuation. (The only difficulty is checking that $w$ is surjective. But this follows fairly easily from [4, Lemma 3].) Hence for each $V_{i}$, we can derive a valuation overring $W_{i}$ of $R$. It is clear that $R^{\prime} \subset \cap W_{i}$. Assume that there is an element $x \in \bigcap W_{i}, x \notin R^{\prime}$. Then the coset $x+N^{\prime} \notin R^{\prime} / N^{\prime}$. Since $R^{\prime} / N^{\prime}$ is the integral closure of $R / N$ in $T(R) / N^{\prime}$, since $T(R) / N^{\prime}$ is a subring of $T(R / N)$, and since $(R / N)^{\prime} \cap T(R) / N^{\prime}=R^{\prime} / N^{\prime}$, we have $x+N^{\prime} \notin(R / N)^{\prime}$. Therefore, $\cap V_{i} \supsetneqq(R / N)^{\prime}$. This contradiction proves that $\bigcap w_{i}=R^{\prime}$. If $w_{i}$ is the valuation corresponding to $W_{i}$, then $\left\{w_{i}\right\}$ satisfies Definition 2.1 for $R^{\prime}$. Q.E.D.

COROLlARY 2.3. If $R$ is a Noetherian ring, then $R^{\prime}$ is a Krull ring. 
THEOREM 2.4. If $R$ is a Noetherian ring and if $P$ is a prime ideal of $R$, then there are only finitely many prime ideals of $R^{\prime}$ that lie over $P$.

Proof. The proof is somewhat similar to the proof of Theorem 2.3 (first divide out the nilradical, then pass to the domain case and use $[8,(33.10)])$. We will not present the details.

Corollary 2.3 and Theorem 2.4 extend the first two parts of $[8,(33.10)]$ to rings with zero divisors.

3. The main theorem. Let $S[X]$ be the polynomial ring in one indeterminate over the ring $S$. For $f \in S[X]$, let $A_{f}$ be the ideal in $S$ generated by the coefficients of $f$. The set $U$ of all $f \in S[X]$ such that $A_{f}=S$ is a regular multiplicative system in $S[X]$. Define $S(X)$ to be the quotient ring $S[X]_{U}$. We will use properties of $A_{f}$ and $S(X)$ without further mention. A general reference for these concepts is [2]. If $I$ is an ideal of $R$, then an element $x$ in $R$ is integral over $I$ in case $x$ satisfies an equation of the form $x^{n}+a_{1} x^{n-1}+\cdots+a_{n}$, where $a_{i} \in I^{i}$. Let $I_{a}$ be the set of all $x$ in $R$ such that $x$ is integral over $I$. The ideal $I_{a}$ is the integral closure of $I$ in $R$. If $\operatorname{Rad} I$ denotes the radical of $I$, then $I \subset I_{a} \subset \operatorname{Rad} I$. If $I$ is a principal regular ideal of $R$, then $I_{a}=I R^{\prime} \cap R[11$, Lemma 2.3]. Let $Z(R)$ be the set of zero divisors in $R$. We need the following concept, which we label as (A).

(A) If $I$ is an ideal of $R$ such that $I \subset Z(R)$, then every finite subset of $I$ has a nonzero annihilator.

Every Noetherian ring satisfies (A) [5, Theorem 82]. If $R$ satisfies (A), then it is easy to see that an overring of $R$ satisfies (A).

We are now ready for the main result of this paper.

Proof of Theorem B. We treat the one- and two-dimensional cases separately.

Case 1. Assume that $\operatorname{dim} R=1$.

There is a one-to-one correspondence between the prime ideals of $R$ consisting entirely of zero divisors and the prime ideals of $T(R)$. Since $R$ and $R^{\prime}$ have the same total quotient ring, there is a one-to-one correspondence between $\left\{Z_{i}\right\}_{i=1}^{g}$, the minimal prime ideals in $R$; and $\left\{Z_{i}^{\prime}\right\}_{i=1}^{g}$, the minimal prime ideals in $R^{\prime}$. In this correspondence $Z_{i}^{\prime} \cap R=Z_{i}$. Thus, we may assume that

$$
R / Z_{i} \subset R^{\prime} / Z_{i}^{\prime} \subset T\left(R / Z_{i}\right) \quad(i=1,2, \ldots, g) .
$$

By $[8,(33.2)], R^{\prime} / Z_{i}^{\prime}$ is a Noetherian domain. Then $R^{\prime} /\left(\bigcap Z_{i}^{\prime}\right)$ is a Noetherian ring [8, (3.16)]. Let $A$ be a regular ideal in $R^{\prime}$ and let $\pi$ be the canonical homomorphism of $R^{\prime}$ onto $R^{\prime} /\left(\bigcap Z_{i}^{\prime}\right)$. Choose $a_{1}, a_{2}, \ldots, a_{n}$ in $A, a_{1}$ regular, such that $\left\{\pi\left(a_{i}\right)\right\}_{i=1}^{n}$ generates $\pi(A)$. If $z \in A$, then $\pi(z)=\sum_{i=1}^{n} \pi\left(c_{i} a_{i}\right)$, where 
$c_{i} \in R^{\prime}$. This implies that $z-\Sigma_{i=1}^{n} c_{i} a_{i}=e \in \bigcap Z_{i}^{\prime}$, and therefore $e$ is a nilpotent element of $R^{\prime}$. We note that every nilpotent element of $T(R)$ is integral over $R$ and is therefore in $R^{\prime}$. In particular, the nilpotent element $e / a_{1}$ is in $R^{\prime}$. Thus, $z-\sum_{i=1}^{n} c_{i} a_{i} \in a_{1} R^{\prime}$. This proves that $A$ has a finite basis.

Case 2. Assume that $\operatorname{dim} R=2$.

By [5, Theorem 7], it suffices to prove that every regular prime ideal $P^{\prime}$ of $R^{\prime}$ has a finite basis, (i.e., is finitely generated).

Part 1. Assume that every regular maximal prime ideal of $R^{\prime}$ is finitely generated and assume that $P^{\prime}$ is a regular height one nonmaximal prime ideal in $R^{\prime}$. Using Theorem 2.4 and Nagata's argument $[8$, p. 121], we may assume that $P^{\prime}$ is the unique prime ideal of $R^{\prime}$ lying over $P^{\prime} \cap R=P$. Let

$$
\left(P^{\prime}\right)=\left\{x \in R^{\prime}: x \text { is regular and } x \notin P^{\prime}\right\} .
$$

Since $R^{\prime}$ is a Krull ring (Corollary 2.3), $R_{\left(P^{\prime}\right)}^{\prime}$ is a discrete rank one valuation ring [7, Proposition 2.5.3]. Choose $x \in R_{\left(P^{\prime}\right)}^{\prime}$ such that $v(x)=1$ ( $v$ is the valuation associated with $\left.R_{\left(P^{\prime}\right)}^{\prime}\right)$. Then $x=b / s$ with $b \in R^{\prime}$ and $s \notin P$, and by Proposition 2.3.2 of [7] we may choose $b$ regular; so $b R_{\left(P^{\prime}\right)}^{\prime}=P^{\prime} R_{\left(P^{\prime}\right)}^{\prime}$. Replace $R$ by the Noetherian ring $R[b]$. We may assume $P^{\prime}$ is the only prime ideal in $R^{\prime}$ lying over $P, b \in P$, and $P^{\prime} R_{\left(P^{\prime}\right)}^{\prime}=P R^{\prime}{ }_{\left(P^{\prime}\right)}=b R^{\prime}{ }_{\left(P^{\prime}\right)}$.

By [9, Proposition 2.13], bR $=\bigcap_{i=1}^{t} P_{i}^{\left(n_{i}\right)}$, where $P_{i}^{\left(n_{i}\right)}=P_{i}^{n_{i}} R_{P_{i}}^{\prime} \cap R^{\prime}$, $\left\{P_{i}\right\}_{i=1}^{t}$ the height one prime ideals of $R^{\prime}$ containing $b$ and $P_{1}=P^{\prime}$. (Note that $R_{P_{i}}^{\prime}$ is the usual localization of $R^{\prime}$ at the prime ideal $P_{i}$ and, in general, is not the same as $R_{\left(P_{i}\right)}^{\prime}$.) Since $P^{\prime\left(n_{1}\right)}$ is a $P^{\prime}$-primary ideal in $R^{\prime}$,

$$
b R_{\left(P^{\prime}\right)}^{\prime} \subset P^{\prime\left(n_{1}\right)} R_{\left(P^{\prime}\right)}^{\prime} \subset P^{\prime} R_{\left(P^{\prime}\right)}^{\prime}=b R_{\left(P^{\prime}\right)}^{\prime},
$$

which implies that $P^{\prime\left(n_{1}\right)}=P^{\prime}$. Thus we can write $b R^{\prime}=P^{\prime} \cap Q_{2}^{\prime} \cap \cdots \cap Q_{t}^{\prime}$, where $Q_{i}^{\prime}$ is a regular height one primary ideal $\not \subset P^{\prime}$. Let $Q^{\prime}=P R^{\prime}: P^{\prime}$. From this point, the proof of (33.12) in [8] carries over to show that $P^{\prime}$ has a finite basis.

Part 2. We prove that every regular maximal ideal of $R^{\prime}$ has a finite basis. Let $M^{\prime}$ be such an ideal. As in Part 1, assume that $M^{\prime}$ is the only prime ideal of $R^{\prime}$ lying over $M^{\prime} \cap R=M$. Then $R^{\prime} / M R^{\prime}=R_{(M)}^{\prime} / M R_{(M)}^{\prime}$. It is sufficient to prove that $M^{\prime} R_{(M)}^{\prime}$ is finitely generated; for then, $M^{\prime} R_{(M)}^{\prime} / M R_{(M)}^{\prime}=$ $M^{\prime} / M R^{\prime}$ is finitely generated, which implies that $M^{\prime}$ has a finite basis. Thus we may assume that $R$ and $R^{\prime}$ have unique regular maximal ideals $M$ and $M^{\prime}$, respectively. Our goal is to prove that $M^{\prime}$ has a finite basis.

If the height of $M^{\prime}$ is 1 , then $R^{\prime}$ is a Krull ring and $R_{\left(M^{\prime}\right)}^{\prime}=R^{\prime}$ is a discrete rank one valuation ring [7, Proposition 2.5.3]. As in the proof of Part 1, $M^{\prime}$ is principal (and is therefore finitely generated).

Assume that $M^{\prime}$ has height 2 . We break the proof of this case into 7 steps. 
Step 1. There exist regular elements $a$ and $b$ in $R$ such that $a$ and $b$ are contained in no common height-one prime ideal of $R$. By the Principal Ideal Theorem and by the fact that $M$ is generated by its set of regular elements [7, Proposition 1.1.2], there exist at least two height-one regular prime ideals of $R$. Let $P$ and $Q$ be two such ideals. Again, $P$ and $Q$ are generated by their respective sets of regular elements. Choose a regular element $a \in P-Q$ and let $\{P=$ $\left.P_{1}, P_{2}, \ldots, P_{t}\right\}$ be the set of minimal prime ideals of the principal ideal $a R$. Choose $c \in Q-\left(\bigcup_{i=1}^{t} P_{i}\right)$ and let $d$ be a regular element in $\left(\bigcap_{i=1}^{t} P_{i}\right) \cap Q$. There is an element $\lambda \in R$ such that $c+d \lambda=b$ is regular [7, Proposition 1.1.2]. Clearly $b \in Q-\bigcup_{i=1}^{t} P_{i}$, and $\{a, b\}$ is the required set.

Step 2. $R^{\prime}[a / b] \cong R^{\prime}[X] /\left((b X-a) R^{\prime}[X]\right)$. Let $\phi: R^{\prime}[X] \rightarrow R^{\prime}[a / b]$ be the natural homomorphism. By [9, Lemma $2.4(5)]$ and by [10, Corollary 7], $\operatorname{ker} \phi$ is generated by the set $\left\{d X-e: d, e \in R^{\prime}, 0 \neq b e=a d\right\}$. From [9, Proposition 2.13], $b R^{\prime}=P_{1}^{\left(n_{1}\right)} \cap P_{2}^{\left(n_{2}\right)} \cap \cdots \cap P_{t}^{\left(n_{t}\right)}$, where $P_{i}$ is a height one regular prime ideal of $R^{\prime}$ and $P_{i}^{\left(n_{i}\right)}=P_{i}^{n_{i}} R_{P_{i}}^{\prime} \cap R^{\prime}$. Consider one of the generators, $d X-e$, of $\operatorname{ker} \phi$. Since $b e=a d \neq 0$, and since $b R^{\prime}$ and $a R^{\prime}$ do not belong to a common height one prime ideal of $R^{\prime}, d \in P_{i}^{\left(n_{i}\right)}$ for each $i$. Therefore $d \in$ $b R^{\prime}$, and writing $d=b s$ with $s \in R^{\prime}$, we see that $s(b X-a)=d X-e$. This proves that $(b X-a) R^{\prime}=\operatorname{ker} \phi$ and $R^{\prime}[X] /\left((b X-a) R^{\prime}[X]\right) \cong R^{\prime}[a / b]$. Denote the ideal $(b X-a) R^{\prime}[X]$ by $I$.

Step 3. $I(R[X])^{\prime} \cap R^{\prime}[X]=I_{a}=\operatorname{Rad} I$. First we show that $\operatorname{Rad} I=$ $\left\{f+c: f \in I\right.$ and $c$ is a nilpotent element in $\left.R^{\prime}\right\}$. Consider $f+c$, where $f \in I$ and where $c$ is nilpotent of index $n$. Then $(f+c)^{n}=f \cdot g+c^{n}=f \cdot g \in I$. On the other hand, let $f \in R^{\prime}[X]$ such that $f^{n} \in I$. In the natural isomorphism from $R^{\prime}[X] / I$ onto $R^{\prime}[a / b], f+I \rightarrow f(a / b)$. But, $f(a / b)$ is nilpotent in $T(R)$ and thus $f(a / b)=c \in R^{\prime}$, since $R^{\prime}$ contains all the nilpotent elements of $T(R)$. However, $c+I=f+I$ (as elements in the ring $R^{\prime}[X] / I$ ) implies that $f-c=$ $f_{0} \in I$. Therefore, $f=f_{0}+c$.

We always have $I \subset I_{a} \subset \operatorname{Rad} I$. Choose $f \in \operatorname{Rad} I$, then $f=f_{0}+c$ where $f \in I$ and $c$ is a nilpotent element of $R^{\prime}$. It follows from [11, Lemma 2.3] that $c \in I(R[X])^{\prime} \cap R^{\prime}[X]=I_{a}$. Thus, $f \in I_{a}$.

Step 4. $R^{\prime}(X)$ is the integral closure of $R(X)$. We always have $T(R)(X) \subset$ $T(R[X])$. Let $f / g \in T(R[X])$, where $f$ and $g$ are in $R[X]$ and $g$ is regular. Then $A_{g}$ is a regular ideal in $R$; for if not, there exists $a \neq 0, a \in R$, such that $a A_{g}=$ 0 (Property (A)), which implies that $g$ is a zero divisor in $R[X]$. Thus, considering $g$ as an element of the ring $T(R)[X]$, we see that $A_{g}=T(R)$; so $f / g \in$ $T(R)(X)$. This proves that $T(R)(X)=T(R[X])$. From [2, Exercise 2, p. 415], $R^{\prime}(X)$ is the integral closure of $R(X)$ in $T(R)(X)=T(R[X])$.

Step 5. If $\left\{P_{1}, P_{2}, \ldots, P_{t}\right\}$ is the set of height-one prime ideals of $R^{\prime}[X]$ such that 
(a) $P_{i} \supset I$,

(b) $P_{i} \subset M^{\prime} R^{\prime}[X]$, then $I R^{\prime}(X)=\left(\bigcap_{i=1}^{t} P_{i}\right) R^{\prime}(X)=\bigcap_{i=1}^{t}\left(P_{i} R^{\prime}(X)\right)$.

Note that there can be only finitely many height-one prime ideals satisfying (a) and (b) [9, Corollary 2.14]. Let $\left\{M_{\beta}\right\}$ be the set of maximal ideals of $R^{\prime}$ and let $P \supset I$ be a height-one prime ideal of $R^{\prime}[X]$. If $P \subset \bigcup_{\beta} M_{\beta} R^{\prime}[X]$, then $P R^{\prime}(X)$ is a prime ideal of $R^{\prime}(X)$. Thus, for some $\beta, P R^{\prime}(X) \subset M_{\beta} R^{\prime}(X)$; hence $P \subset M_{\beta} R^{\prime}[X]$. Assume that $M^{\prime} \neq M_{\beta}$, then $M_{\beta} \subset Z\left(R^{\prime}\right)$. Since $I \subset P, I \subset$ $M_{\beta} R^{\prime}[X]$. Write $b X-a=\Sigma_{i=1}^{s} \alpha_{i} f_{i}$, where $\alpha_{i} \in M_{\beta}$ and $f_{i} \in R^{\prime}[X]$. By Property (A), there exists $c \neq 0$ such that $c$ annihilates $\left\{\alpha_{1}, \alpha_{2}, \ldots, \alpha_{s}\right\}$. Then $c(b X-a)=0$. This contradiction proves that if $P$ is a height-one prime ideal of $R^{\prime}[X], P \supset I$, and $P \subset \bigcup_{\beta} M_{\beta} R^{\prime}[X]$; then $P \subset M^{\prime} R^{\prime}[X]$.

From Step 4, $R[X] \subset R^{\prime}[X] \subset(R[X])^{\prime} \subset R^{\prime}(X)$. Let $J=\bigcap_{i=1}^{t} P_{i}$. It follows from Step 3 that $I R^{\prime}(X)=I(R[X])^{\prime} R^{\prime}(X)=I_{a} R^{\prime}(X)=J R^{\prime}(X)=\bigcap_{i=1}^{t}\left(P_{i} R^{\prime}(X)\right)$.

Step 6. If $p$ is a minimal prime ideal of $R^{\prime}(X)$, then $T(R[X] / \mathfrak{p} \cap R[X])=T\left(R^{\prime}[X] / \mathfrak{p} \cap R^{\prime}[X]\right)=T(R(X) / \mathfrak{p} \cap R(X))=T\left(R^{\prime}(X) / \mathfrak{p}\right)$.

This holds since $p$ intersected with any of the rings in question is a prime ideal consisting entirely of zero divisors.

Step 7. $M^{\prime}$ is finitely generated. From Step $5, I R^{\prime}(X)=\bigcap_{i=1}^{t}\left(P_{i} R^{\prime}(X)\right)$ where each $P_{i}$ is a height-one prime ideal of $R^{\prime}[X]$ such that $P_{i} \subset M^{\prime} R^{\prime}[X]$. For each $i$ fix $\mathfrak{p}_{i}$, a minimal prime ideal of $R^{\prime}(X)$ such that $P_{i} R^{\prime}(X) \supsetneqq p_{i}$. Then $P_{i} R^{\prime}(X) \cap R(X) \supsetneqq p_{i} \cap R(X)$. Let $S=R(X) /\left(\mathfrak{p}_{i} \cap R(X)\right)$ and $S^{*}=R^{\prime}(X) / \mathfrak{p}_{i}$. Then $S$ and $S^{*}$ are integral domains such that

(i) $S \subset S^{*} \subset T(S)$,

(ii) $S^{*}$ is integral over $S$,

(iii) $S$ is a Noetherian domain,

(iv) $\operatorname{dim} S=\operatorname{dim} S^{*}=2$.

Let $P_{i}^{*}$ be the height-one prime ideal of $S^{*}$ corresponding to $P_{i} R^{\prime}(X)$. By [8, (33.10)] and the fact that $S^{*} \subset S^{\prime}, S^{*} / P_{i}^{*}$ is an almost finite integral extension of $S /\left(P_{i}^{*} \cap S\right)$, and $\operatorname{dim} S /\left(P_{i}^{*} \cap S\right)=1$. By the Krull-Akizuki Theorem [8, (33.2)], $S^{*} / P_{i}^{*}=R^{\prime}(X) /\left(P_{i} R^{\prime}(X)\right)$ is a Noetherian ring. By [8, (3.16)], $R^{\prime}(X) /\left(\bigcap P_{i} R^{\prime}(X)\right)=R^{\prime}(X) / I R^{\prime}(X)$ is a Noetherian ring. Thus $M^{\prime} R^{\prime}(X) / I R^{\prime}(X)$ is finitely generated. This implies that $M^{\prime} R^{\prime}(X)$ has a finite basis. If $\left\{f_{i}\right\}_{i=1}^{r}$ are elements of $R^{\prime}[X]$ that generate $M^{\prime} R^{\prime}(X)$, then the coefficients of the $f_{i}$ form a generating set for $M^{\prime}$. Thus, $M^{\prime}$ has a finite basis. Q.E.D.

We close this paper with a result that shows how to construct a Noetherian ring $R$ of any positive dimension such that $R^{\prime}$ is non-Noetherian. Hence, Theorem A cannot be generalized by replacing "domain" with "ring". 
Proposition 3.1. Let $D$ be an n-dimensional local (Noetherian) domain, $n \geqslant 2$. Assume that $P$ is a height-one prime ideal of $D$ of depth $n-1$, and assume that $A$ is a P-primary ideal distinct from $P$. If $R=D / A$, then $R^{\prime}$ is a non-Noetherian ring of dimension $n-1$.

Proof. Assume that $R^{\prime}$ is a Noetherian ring. Choose a regular nonunit $b$ in the Jacobson radical of $R$, and hence in the Jacobson radical of $R^{\prime}$. The nilradical $N^{\prime}$ of $R^{\prime}$ is a nonzero ideal in $R^{\prime}$. Since $R^{\prime}$ contains all the nilpotent elements of $T(R), b N^{\prime}=N^{\prime}$. By Nakayama's Lemma [5, Theorem 78], $N^{\prime}=$ (0), a contradiction. Therefore, $R^{\prime}$ is a non-Noetherian ring. Clearly $\operatorname{dim} R^{\prime}=$ $n-1$. Q.E.D.

\section{REFERENCES}

1. E. Davis, Overrings of commutative rings. II, Trans. Amer. Math. Soc. 110 (1964), 196-212. MR 28 \#111.

2. R. Gilmer, Multiplicative ideal theory, Dekker, New York, 1972.

3. R. Gilmer and J. A. Huckaba, $\Delta$-rings, J. Algebra 28 (1974), 414-432.

4. J. A. Huckaba, On valuation rings that contain zero divisors, Proc. Amer. Math. Soc. 40 (1973), 9-15. MR 47 \#683.

5. I. Kaplansky, Commutative rings, Allyn and Bacon, Boston, Mass., 1970. MR 40 \#234.

6. M. Larsen and P. McCarthy, Multiplicative theory of ideals, Academic Press, New York and London, 1971.

7. J. Marot, Extension de la notion d'anneau de valuation, Dept. Math. Faculté des Sciences de Brest, 1968.

8. M. Nagata, Local rings, Interscience Tracts in Pure and Appl. Math., no. 13, Interscience, New York and London, 1962. MR 27 \#5790.

9. L. J. Ratliff, Jr., On prime divisors of the integral closure of a principal ideal, J. Reine Angew. Math. 255 (1972), 210-220. MR 47 \#200.

10. - Conditions for $\operatorname{Ker}(R[X] \rightarrow R[c / b])$ to have a linear bass, Proc. Amer. Math. Soc. 39 (1973), 509-514. MR 47 \#4989.

11. Locally quasi-unmixed Noetherian rings and ideals in the principal class, Pacific J. Math. 52 (1974), 185-205.

\section{DEPARTMENT OF MATHEMATICS, UNIVERSITY OF MISSOURI, COLUMBIA, MISSOURI 65201}

